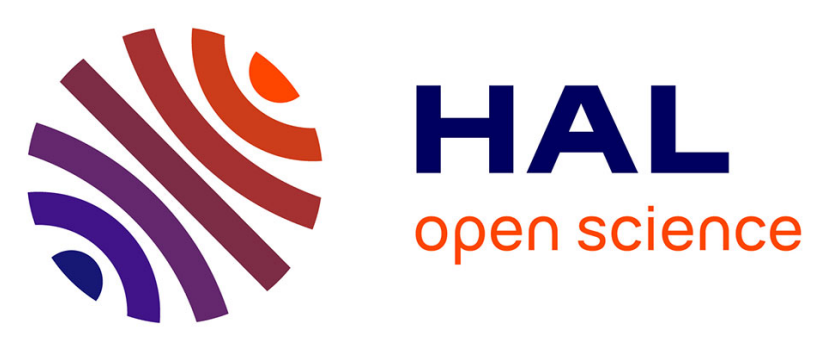

\title{
Évolutions conjointes de la situation scolaire et de l'orientation en France : quelques repères pour une histoire des services d'orientation
}

Régis Ouvrier-Bonnaz

\section{- To cite this version:}

Régis Ouvrier-Bonnaz. Évolutions conjointes de la situation scolaire et de l'orientation en France : quelques repères pour une histoire des services d'orientation. L'Orientation scolaire et professionnelle, 2019, 48/2, pp.211-230. 10.4000/osp.10736 . hal-03450777

\section{HAL Id: hal-03450777 \\ https://hal-cnam.archives-ouvertes.fr/hal-03450777}

Submitted on 26 Nov 2021

HAL is a multi-disciplinary open access archive for the deposit and dissemination of scientific research documents, whether they are published or not. The documents may come from teaching and research institutions in France or abroad, or from public or private research centers.
L'archive ouverte pluridisciplinaire $\mathbf{H A L}$, est destinée au dépôt et à la diffusion de documents scientifiques de niveau recherche, publiés ou non, émanant des établissements d'enseignement et de recherche français ou étrangers, des laboratoires publics ou privés.

$$
\text { Copyright }
$$




\section{O.S.P.}

\section{L'orientation scolaire et professionnelle}

$48 / 2$ | 2019

Regards sur l'histoire de l'orientation en France

\section{Évolutions conjointes de la situation scolaire et de l'orientation en France : quelques repères pour une histoire des services d'orientation}

Joint developments of school situation and vocational gui-dance in France: some benchmarks for an history of guidance services

\section{Régis Ouvrier-Bonnaz}

\section{OpenEdition \\ Journals}

Édition électronique

URL : https://journals.openedition.org/osp/10736

DOI : 10.4000/osp.10736

ISSN : 2104-3795

\section{Éditeur}

Institut national d'étude du travail et d'orientation professionnelle (INETOP)

\section{Édition imprimée}

Date de publication : 1 juin 2019

Pagination : 211-230

ISSN : 0249-6739

Ce document vous est offert par Conservatoire national des arts et métiers (Cnam)

\section{e cnam}

\section{Référence électronique}

Régis Ouvrier-Bonnaz, «Évolutions conjointes de la situation scolaire et de l'orientation en France : quelques repères pour une histoire des services d'orientation ", L'orientation scolaire et professionnelle [En ligne], 48/2 | 2019, mis en ligne le 01 juin 2021, consulté le 26 novembre 2021. URL : http:// journals.openedition.org/osp/10736; DOl : https://doi.org/10.4000/osp.10736 


\section{Évolutions conjointes de la situation scolaire et de l'orientation en France : quelques repères pour une histoire des services d'orientation}

Joint developments of school situation and vocational gui- dance in France: some benchmarks for an history of guidance services

Régis Ouvrier-Bonnaz

\section{Introduction}

1 Comme l'indique Michel Huteau (1999), si l'histoire des services d'orientation commence en France à la fin des années 1910, à l'issue de la Première Guerre mondiale, l'existence de débats sur la nécessité d'asseoir l'orientation sur le plan institutionnel prend forme dans les changements sociaux et économiques de la fin du XIX ${ }^{\mathrm{e}}$ siècle. Pour lui, «l'histoire de l'orientation est en fait la résultante de plusieurs histoires qui interagissent: l'histoire économique et sociale, l'histoire de la scolarisation et de l'appareil scolaire, l'histoire des structures administratives, l'histoire de la psychologie, l'histoire des idées et celle des pratiques » (p. 13).

2 La dégradation des formes d'accès au métier et des conditions du travail ouvrier, à l'aube du XXe siècle, fait de la formation professionnelle des jeunes une question centrale. Dans ce contexte, l'idée d'orientation qui commence à se développer répond au souci de donner aux jeunes les postes qui correspondent le mieux à leurs aptitudes. L'idée de mesurer les caractéristiques psychologiques en vue d'organiser la répartition des individus dans le marché du travail n'est pas nouvelle, mais l'orientation lui confère, dans les premières années du $\mathrm{XX}^{\mathrm{e}}$ siècle, une nouvelle forme de légitimité scientifique en la considérant comme une application de la psychologie et de ses outils : 
les tests. Dans cette volonté de classification, le concept d'aptitude comme disposition naturelle et sa mesure sont donnés comme centraux.

\section{La mesure des aptitudes : une préoccupation commune au monde du travail et à l'école}

3 Le premier cadre conceptuel de l'orientation professionnelle est connu : les individus se différencient par des aptitudes héréditaires étroitement spécifiques, les métiers sont des entités stables exigeant des aptitudes très spécifiques. L'objet des applications de la psychologie dans le domaine de l'orientation est de favoriser l'ajustement des aptitudes du sujet à celles exigées par les métiers et de contribuer ainsi à ce que chacun soit à sa place (Huteau \& Lautrey, 1979). La même préoccupation est présente dans l'espace scolaire de ce début de siècle, comme en témoigne la préface d'Alfred Binet dans L'Année psychologique en 1908 à propos des études à l'origine de la construction de l'échelle métrique de l'intelligence : «La portée de ces études dépasse la pédagogie, car nous envisagerons en particulier les aptitudes relativement aux métiers qu'ils se destinent à exercer... nous pensons que dans ce domaine, on peut faire beaucoup pour améliorer les rapports des ouvriers et des patrons, et pour aider à la solution de la question ouvrière, en avertissant les enfants, dès l'école, des professions pour lesquelles ils sont les plus aptes, et en diminuant ainsi, par cette prophylaxie professionnelle le nombre de sujets mal adaptés, qui deviennent nécessairement des déclassés, des malheureux ou des insoumis ${ }^{1}$.» (p. V-VI).

Jean Guichard (1987) relève à propos de ce texte « qu'il dit en peu de mots l'essentiel de la préoccupation politique et sociale qui constitue le cadre idéologique où se formulent les premiers discours scientifiques relatifs à l'enfance inadaptée (ou à l'orientation) sans ces préoccupations sociales, politiques et morales de la fin du XIX siècle, ces discours scientifiques n'auraient pu se formuler : les problèmes à résoudre n'étant tout simplement pas visibles, pas apercevables en tant que problèmes sociaux, et donc pas constituables en questions scientifiques. Mais, d'autre part, les discours tenus sur ces questions, la manière scientifique de les aborder vont nécessairement porter la marque du cadre idéologique qui en a permis la formulation ${ }^{2}$.»

5 Le cadre qui fonde l'accord à propos de l'orientation professionnelle prend forme dans l'idée d'une société idéale organisée selon les lois de la Raison où chacun trouverait sa place. Il dessine les contours d'un espace susceptible d'être isolé comme un champ disciplinaire autonome où l'étude des aptitudes apparaît comme le paradigme dominant. Le mot d'aptitude a été employé dans le domaine des sciences humaines et sociales avec des sens différents. Cette diversité a contribué à rendre confus des débats qui n'ont pas toujours respecté les règles des discussions scientifiques. Le mot recouvre une notion ancienne dont l'histoire est de beaucoup antérieure à son application dans le champ de l'orientation. Au XVI ${ }^{\mathrm{e}}$ siècle, aptitude, terme purement juridique prend le sens d'une disposition naturelle de caractère divin qui, cependant, ne confère pas de supériorité de rang, le pouvoir restant lié à la naissance. Le mot est peu usité jusqu'au XVIII ${ }^{e}$ siècle où, sous l'influence de la pensée des Lumières, il est référé aux notions de mérite et de responsabilité individuelle pour s'articuler, à la fin du siècle, au début de la révolution industrielle et technique, à l'activité pratique de l'homme. C'est à partir de cette articulation, à un moment où le privilège de la naissance est contesté, dans la logique de la Révolution de 1789 , que la société industrielle et commerciale de la 
première moitié $d u x x^{e}$ siècle tente d'asseoir sa légitimité sur une idéologie justificatrice des inégalités. Les capacités individuelles, jugées comme essentielles et héréditaires, justifient la place de chacun dans la société. Pour Noëlle Bisseret (1974), les inégalités sociales relèvent alors d'une nature biologique irréductible et déterminante.

6 À une période où se développe de manière importante la crise de l'apprentissage et de l'accès au métier, la mesure scientifique des aptitudes apparaît comme un progrès de la raison. Dans le même temps avec l'évolution industrielle, une opposition grandissante s'installe entre des activités professionnelles se limitant à l'utilisation de la seule force physique des ouvriers et des activités de plus en plus spécialisées demandant un niveau de qualification que le système de formation n'est pas en mesure d'offrir (Prost, 1968). Deux conceptions de la qualification entrent en concurrence. L'une est caractérisée par la simplification et la fragmentation des tâches professionnelles. L'autre, représentée par l'enseignement professionnel dès sa création, insiste sur l'importance des acquisitions théoriques et des phénomènes de transfert de ces acquis. Les luttes d'influence entre l'État, et au sein même de l'État, entre les ministères de l'Instruction publique et du Commerce, le patronat, les pouvoirs politiques locaux vont déterminer le rythme de développement de l'enseignement professionnel et les modes d'accès à cet enseignement.

\section{L'installation et le développement d'un enseignement professionnel et la question de l'orientation}

7 Dès 1829 , des cours de deux ans portant sur les sciences et leur application à l'industrie et sur la théorie du commerce existaient dans les collèges, mais c'est la loi du 2 octobre 1863 sur l'enseignement secondaire spécial qui tentera d'installer un enseignement spécifique. Dans le cadre des discussions qui participent à la création de cet enseignement, le terme d'aptitude est utilisé pour la première fois en 1852, dans un texte à propos de la proposition Fortoul qui institue deux voies de formation après la classe de troisième des lycées (Isambert-Jamati, 1972). Cet enseignement spécial n'est pas réellement professionnel, mais il doit servir d'introduction à l'entrée dans les professions (Lelièvre, 1990). Le souci de lier la théorie et la pratique est lié à la conjoncture économique et à la réalité technique de l'époque.

Victor Duruy, ministre de l'Instruction publique de 1863 à 1869, dans une lettre adressée aux recteurs sur l'organisation de l'enseignement spécial, le rappelle sans ambiguïté : "Dans la lutte pacifique - mais redoutable - qui est engagée entre les peuples industrieux, le prix n'est pas réservé à celui qui disposera de plus de bras ou de capitaux, mais à la nation au sein de laquelle les classes laborieuses auront le plus d'intelligence et de savoir. La science met chaque jour au service de l'industrie des agents nouveaux qui la secondent. Voilà pourquoi le progrès industriel est aujourd'hui étroitement lié au progrès scolaire ${ }^{3}$.» Le développement de l'enseignement spécial répond à une double préoccupation : la nécessité de repenser l'organisation du travail et la volonté d'élever tout le monde sans déclasser personne. Malgré un succès indéniable, cet enseignement va progressivement évoluer pour se transformer en 1902 (décret du 31 mai) en un secondaire moderne qui ne présente plus de caractère professionnel. 


\section{L'enseignement primaire et le développement de la formation professionnelle}

9 Face aux réticences à rattacher un enseignement professionnel spécifique au lycée, c'est du côté de l'enseignement primaire qu'il va se développer. La loi du 11 décembre 1880 institue un enseignement professionnel court: les écoles manuelles d'apprentissage relevant du ministère du Commerce et les classes d'enseignement des écoles primaires supérieures (EPS) placées sous la tutelle du ministère de l'Instruction publique. Les mieux équipées des EPS passeront en 1892 (décret du $1^{\mathrm{er}}$ juin) sous la responsabilité du Premier ministère pour prendre le nom d'écoles pratiques de commerce et d'industrie (EPCI). Très vite ce partage de responsabilité débouche sur un partage de nature idéologique (Caroff, 1987). Au ministère de Commerce et de l'Industrie, les écoles préparant à un métier ; à l'Instruction publique et aux Beaux-Arts, celles qui préparent à l'apprentissage. Cette distinction révèle un certain nombre de tensions qui sont encore vivaces dans l'enseignement professionnel et technologique : tensions entre l'atelier et l'école, le concret et l'abstrait, le général et le particulier, la théorie et la pratique, l'esprit et la main. Le débat d'idées que l'on retrouvera au moment de définir les certifications professionnelles oppose les adeptes d'une formation professionnelle assez étroitement calquée sur l'organisation du travail aux tenants d'une conception plus large de la formation qui s'intègre dans un projet éducatif plus large.

10 Toutes ces tensions constituent, de fait, autant de critères de discrimination des enseignements, des élèves, de l'apprentissage et de l'orientation professionnelle (Fontègne, 1921). En réalité, aucune de ces écoles ne va former de simples ouvriers. Comme les Écoles nationales professionnelles (ENP) créées en 1881 mais dont la première ouvrira seulement en 1886 et les écoles techniques de la ville de Paris ouvertes un peu plus tard, elles forment l'élite de la classe ouvrière. Dans tous ces projets, si l'orientation professionnelle est présente, de manière plus ou moins explicite, elle ne fait pas l'objet d'un traitement spécifique. Au tournant du $\mathrm{xx}^{\mathrm{e}}$ siècle, la pénurie de main-d'œuvre s'accentue et la crise de l'apprentissage demeure.

\section{La crise de l'apprentissage et les premières formes d'orientation dans l'école : les cours de préapprentissage}

11 Le débat sur l'apprentissage est ancien mais il est relancé à la fin du XIX siècle par une industrialisation massive conduite dans un contexte où domine un libéralisme qui aggrave les conditions de vie et d'emploi des ouvriers et fragilise les plus démunis, en particulier les enfants. Dans ce contexte, l'orientation professionnelle et l'apprentissage sont considérés comme des éléments pouvant permettre une meilleure intégration et une plus grande justice sociales. Le mouvement d'orientation naît en dehors de l'école et va se développer à sa périphérie à une exception près, rarement évoquée, qui peut être considérée comme la première forme de l'orientation professionnelle dans les milieux de formation: les cours de préapprentissage. Elle est marquée par les lois de laïcisation de l'école de 1904. En 1911, lors du congrès de Roubaix consacré à l'apprentissage, organisé par l'Association française pour le 
développement de l'enseignement techniques (Afdet) créée au début du siècle, le préapprentissage est défini comme une période destinée à faire naître chez l'enfant le choix d'une profession. L'articulation entre l'orientation professionnelle et la formation professionnelle est ainsi clairement affirmée (Martin, 2002). Deux expériences vont porter les discussions autour de cette notion :

- l'atelier de préapprentissage patronal ouvert en 1907 par Kula, entrepreneur parisien de couverture-plomberie, initiative soutenue par les milieux patronaux ;

- les classes de préapprentissage ouvertes en 1911 dans quatre écoles primaires parisiennes à l'initiative de A. Jully, inspecteur principal de l'enseignement du travail manuel des écoles primaires de Paris, avec le soutien de la Ligue de l'enseignement.

Sur le plan institutionnel, la création de ces cours de préapprentissage apparaît d'abord comme une des manifestations de la concurrence entre les ministères du Commerce et de l'Instruction publique (Briand, 1989). Dans les faits, elle est l'expression concrète des débats sur l'évolution de la prolongation de la scolarité obligatoire et la création d'un enseignement technique de type scolaire. Ces initiatives, que l'on peut aussi analyser à l'aune des discussions plus générales sur l'apprentissage professionnel qui aboutira à la loi Astier de 1919, n'auront pas de suite sur le plan législatif. N'ayant pas trouvé de réponse satisfaisante, cette question du préapprentissage réapparaîtra régulièrement à chaque moment de crise de l'école pour interroger la liaison formation/emploi et l'orientation.

\section{La loi Astier de 1919 et la reconnaissance de l'orientation professionnelle}

13 La loi qui est votée par le Parlement dans sa séance du 4 juillet 1919, souvent associée à la seule création du certificat d'aptitude professionnelle (CAP) est l'aboutissement de quinze années de discussions d'un projet de loi déposé en 1905 par le ministre du Commerce de l'époque. Celle-ci visait à organiser l'enseignement technique public sous l'autorité de son ministère, à établir le contrôle de l'état sur l'enseignement technique privé et à instituer des cours professionnels obligatoires pour les jeunes de moins de 18 ans travaillant dans le commerce et l'industrie. La loi de 1919 reprend ces dispositions dans leurs grandes lignes et les codifie mais elle innove en édictant une double obligation concernant les cours professionnels. Les communes sont tenues de créer les cours et de subvenir à leur fonctionnement et le suivi des cours est rendu obligatoire. Elle précise aussi que des écoles de métiers pourront être créées par les chambres de commerce ou des associations professionnelles. Aucune disposition de cette loi ne se relie directement à l'orientation professionnelle.

Cependant comme le signale Caroff (1987, p. 48-69) dans son étude sur l'organisation de l'orientation des jeunes en France, poussée par des initiatives locales de plus en plus nombreuses, au début des années 1920, l'orientation professionnelle devient en moins de trois ans une réalité nationale s'incarnant dans les institutions. Cette évolution est largement soutenue par tout un courant scientifique lié à la création et au développement de la psychotechnique et par la création en 1921, à l'initiative de Henri Piéron, d'un Institut de psychologie qui comprend une section d'orientation et sélection professionnelles. Un changement institutionnel va être déterminant au niveau gouvernemental : la création en 1920 pour la première fois au sein du ministère 
de l'Instruction publique et des Beaux-Arts d'un sous-secrétariat d'État à l'Enseignement technique confiant à ce ministère la direction de l'enseignement technique rattachée auparavant au ministère du Commerce et de l'Industrie. Dès lors, si la rationalisation du placement des jeunes est reconnue comme une nécessité, elle ne subordonne pas l'orientation aux seuls impératifs économiques en distinguant l'orientation du placement. Pour Edmond Labbé, directeur de l'enseignement technique de 1920 à 1933, l'orientation participe à la réalisation de la justice sociale en mettant chacun à sa place et c'est pourquoi elle doit être conçue comme une question d'éducation.

Le décret du 26 septembre 1922 qui marque la création des offices d'orientation professionnelle et les place sous la responsabilité du sous secrétariat à l'Enseignement technique repose sur un compromis et la mise en ordre administrative ne change guère la réalité (Prost, 1996). Le service d'orientation reste facultatif et les offices d'orientation continuent d'être financés par des acteurs privés, des entreprises ou des organismes ou collectivités publiques (chambre de métiers, commune, département ...). Dans ce contexte, la création en 1928 de l'Institut national d'orientation professionnelle (Inop) par Henri Piéron, Henri Laugier et Julien Fontègne, si elle permet de poser la question de la formation des personnels des offices, répond plus à une préoccupation scientifique qu'à une réelle demande sociale ${ }^{4}$. L'inauguration de cet institut créé avec le soutien actif du sous-secrétariat d'État à l'enseignement technique, donne à Labbé l'occasion de préciser le lien entre l'enseignement technique et l'orientation. Celle-ci « est un élément primordial de l'enseignement technique ou, pour mieux dire, elle en est la base ${ }^{5}$ ». La possibilité de faire appel à un personnel qualifié va renforcer le rôle des offices d'orientation. En 1932, l'Inop participe, au côté d'autres associations, à la création du Bureau universitaire de statistique (BUS). Cet organisme se donne pour objectif d'établir une vaste documentation scolaire et professionnelle pour lutter contre le chômage intellectuel et favoriser une meilleure intégration des étudiants diplômés sur le marché du travail. Les initiatives concernant l'orientation sont nombreuses, mais son développement ne s'inscrit pas dans une conception globale. Le statut des offices d'orientation est incertain, l'Inop a un statut d'établissement privé, le BUS a un statut de type associatif, un important effort d'institutionnalisation reste donc à faire. Ce sera l'œuvre du gouvernement de Front populaire.

\section{Le gouvernement de Front populaire et la création des services d'orientation}

16 Le gouvernement du Front populaire issu des élections législatives du 5 mai 1936, soucieux de justice sociale et de progrès économique, va jeter les bases d'un véritable service d'orientation. L'une des premières décisions du ministère de l'Éducation nationale dirigé par Jean Zay (1904-1944) est de porter l'âge de la scolarité obligatoire à 14 ans. Jean Zay a devant lui la durée. Il va rester en poste de juin 1936 à la déclaration de la guerre en septembre 1939. Dès son arrivée, il prépare un projet d'ensemble de refonte du système éducatif qui s'articule sur la création d'un véritable second degré qui met fin au cloisonnement des différents ordres d'enseignement. Pour cela, il propose de transformer les trois enseignements parallèles (classique, moderne et technique) en trois sections d'un même enseignement. Pour assurer l'orientation des élèves entre ces trois sections, le projet prévoit de transformer la classe de sixième en 
véritable classe de détermination. Les oppositions à ce projet sont nombreuses et les premières classes d'orientation (arrêté du 22 mai 1937) vont très vite se réduire à un seul trimestre sans latin. C'est du côté du développement de la formation professionnelle que le mouvement de l'orientation prend forme pour faire face à la crise de l'emploi. La pénurie de main-d'œuvre qualifiée est un des problèmes majeurs auquel le gouvernement de Front populaire doit s'attaquer. Le moyen d'améliorer l'accès à l'emploi et de lutter contre le chômage réside dans l'amélioration de la formation professionnelle. Dans ce domaine, la loi Astier n'a pas donné tous les résultats attendus. Deux textes de loi vont être adoptés, tous les deux lient l'orientation à la formation professionnelle, ce que la loi Astier n'avait pas fait :

- la loi du 10 mars 1937 portant organisation de l'apprentissage dans les entreprises artisanales connue sous le nom de loi Walter-Paulin ;

- le décret de loi du 24 mai 1938 relatif à l'orientation et à la formation professionnelle dans le commerce et l'industrie.

17 Le second texte précise qu'aucun jeune de moins de 17 ans ne peut être employé sans un certificat d'orientation professionnelle indiquant d'éventuelles contre-indications à l'exercice du métier choisi. Cette disposition implique de créer un nouveau service. En 1939, deux décrets fixent les modalités de création et de fonctionnement des centres d'orientation professionnelle. Un secrétariat d'orientation professionnelle départemental ou interdépartemental est institué, animé par un secrétaire d'orientation professionnelle. Il est soumis à l'autorité du préfet et entre dans les attributions des inspecteurs d'académie. D'autres dispositions législatives préciseront le lien avec le système scolaire et l'obligation faite aux directeurs d'école de collaborer avec ces centres, mais aussi avec les services de placement qui sont tenus d'adresser au secrétaire d'orientation l'état du marché du travail local.

Les bases sont posées mais faute de moyens, le dispositif va avoir du mal à se développer. La période de la guerre et l'Occupation sont des moments difficiles pour l'orientation malgré la volonté affichée d'un homme: Hippolyte Luc, directeur de l'enseignement technique depuis 1933. Maintenu en poste pendant la guerre, il s'efforce de faire paraître certains textes d'application du décret-loi du 24 mai 1938. Sa tâche est difficile du fait de l'éclatement de la formation professionnelle tiraillée entre l'enseignement technique qui dépend du secrétariat général de l'instruction publique et le service de formation des jeunes rattaché au secrétariat général de la jeunesse et au commissariat des chantiers de jeunesse. Le monopole de l'Institut d'étude du travail et d'orientation professionnelle (Inetop) est contesté, mais un diplôme d'État délivré par cet institut est créé qui donne à ses titulaires le titre de conseiller d'orientation professionnelle ${ }^{6}$. Un Centre national de documentation professionnelle y est créé (arrêté du 4 avril 1943) dont la mise en place sera effective en 1945 sous l'appellation de Centre d'études et de recherches documentaires de l'enseignement technique (Cerdet).

Parallèlement, la décision est prise de créer de nouveaux centres de formation de conseillers d'orientation professionnelle. L'Institut de biométrie humaine et d'orientation professionnelle (Ibhop) de Marseille est fondé par décret du 15 avril 1943. Il sera ouvert après la fin de la guerre comme institut universitaire placé sous le contrôle scientifique de la Faculté de médecine. Il est dirigé, sous l'autorité du recteur de l'académie, par un professeur d'université et géré par un conseil d'administration. À la Libération, les services d'orientation vont connaître un nouvel essor et ce modèle servira d'exemple pour la création des centres de formation qui accompagneront leur 
développement. C'est également pendant la période de la guerre que les bases d'un enseignement professionnel de masse sont jetées et que sont créés les centres de formation professionnels (CFP). Ces centres transformés en centres d'apprentissages publics (loi du 21 février 1949) sont à l'origine de la création de l'enseignement professionnel de type scolaire de la seconde moitié $\mathrm{du} \mathrm{xx}^{\mathrm{e}}$ siècle dont les collèges d'enseignement technique (CET), ouverts en 1960 par transformation des centres d'apprentissage, constituent le socle.

\section{L'institutionnalisation des services d'orientation au sein de l'Éducation nationale et la fonctionnarisation de ses personnels}

20 La période de l'après-guerre est marquée par une remise en cause globale du système d'enseignement. Une commission connue sous le nom de ses deux présidents successifs, Langevin-Wallon, élabore un plan considéré comme l'aboutissement de deux courants de pensée de l'entre-deux-guerres : l'école unique pour les structures et l'école nouvelle pour la pédagogie (Prost, 1968). Ce plan qui prévoyait un degré d'enseignement intermédiaire (11-15 ans) baptisé cycle d'orientation et la création d'un nouveau corps de psychologues scolaires à côté des conseillers d'orientation professionnelle ne verra jamais le jour. Henri Piéron, un des deux vice-présidents de la commission, justifiait dans un numéro du Bulletin de l'Institut national d'orientation professionnelle (Binop) de 1945, l'existence de deux corps en charge de l'adaptation et de l'orientation des élèves : «Dans le projet de réforme de l'enseignement préparé par la commission Langevin, une place importante doit être donnée à l'orientation scolaire, afin d'approprier aux aptitudes de chacun la formation éducative, prélude obligatoire à la formation professionnelle. Les liens sont évidemment étroits entre cette orientation éducative et l'orientation professionnelle, car diriger un enfant après l'éducation générale commune du premier degré vers des branches théoriques et pratiques au second degré de la scolarité, c'est bien l'embrancher dans une voie qui comporte des catégories de professions entièrement différentes dans un cas ou dans l'autre. Mais cette orientation initiale n'envisage que l'aptitude à profiter d'enseignement d'une certaine nature et d'un certain niveau, sans se préoccuper des métiers et des carrières fort diverses qui pourront être embrassés plus tard. Le problème de l'orientation professionnelle proprement dite se pose au moment où doit se faire la préparation éducative au métier ${ }^{7}$ ».

21 Ce texte, en distinguant deux fonctions, suggère l'existence de deux corps de personnels susceptibles de les supporter : les psychologues scolaires dans l'école et les conseillers d'orientation professionnels au croisement de l'école et du monde du travail. Selon Caroff (1987), cette séparation est conforme aux intentions affichées en 1938 dont plusieurs dispositions vont être modifiées. En particulier, le secrétariat d'orientation professionnelle, considéré comme l'instance de coordination des actions des centres obligatoires d'orientation, est déplacé du niveau départemental au niveau académique. Au même moment, les conseils généraux contestent les charges budgétaires qui reviennent au département en matière de gestion de centres d'orientation aux statuts divers. Le gouvernement, sur une proposition de Rachel Lempereur, députée socialiste du Nord, présidente de la commission de l'Éducation 
nationale de l'Assemblée nationale, réorganise les services d'orientation (loi du 24 mai 1951). Le texte définitif de cette loi prévoit trois mesures :

- la création d'une inspection de l'orientation professionnelle à la charge de l'État au niveau de chaque académie ;

- les centres obligatoires d'orientation professionnelle sont transformés en centres publics d'orientation professionnelle introduisant ainsi une distinction avec les centres privés ;

- la rémunération du directeur, des conseillers et du personnel administratif est à la charge de l'État.

Il faudra attendre encore quatre ans (décret du 10 octobre 1955) pour que le statut des centres soit défini et cinq ans pour que le statut du personnel soit précisé. Dès lors, la structure des services d'orientation, telle qu'elle se présente encore aujourd'hui, est posée et l'évolution des services va être essentiellement qualitative pour suivre l'évolution et la restructuration du système scolaire. La volonté d'installer une école unique, déjà en germe à la fin du premier conflit mondial, conduit à substituer progressivement à une école par ordre (primaire, secondaire, technique et professionnel) une école par degrés (école élémentaire, collège, lycée). Cette réforme structurelle d'envergure est l'œuvre de la V ${ }^{\mathrm{e}}$ République de la fin des années 1950 et du début des années 1960. En 1956, l'examen d'entrée en classe de sixième est supprimé et en 1959, la réforme dite Berthoin (ordonnance du 6 janvier 1959), du nom du ministre de l'Éducation nationale qui l'a préparée, porte la scolarité obligatoire à 16 ans pour les enfants ayant atteint l'âge de 6 ans au $1^{\text {er }}$ janvier 1959. Pour accueillir les élèves jusqu'à 16 ans, il faut créer une structure unique.

Un décret du 3 août 1963 institue le collège d'enseignement secondaire (CES) qui accueille les enfants de 11 à 15 ans dans trois filières différentes avec leur encadrement, leur pédagogie et leurs débouchés différents. Dans les faits, cette organisation oppose un enseignement d'excellence (la voie I), qui vise à élargir le recrutement des «élites scolaires » à la prolongation de la scolarité pour la grande masse des élève (la voie II et III). En 1961, dans cette logique de développement de la scolarisation, les personnels des centres d'orientation professionnelle, devenue scolaire et professionnelle (COSP) deux ans auparavant, deviennent des conseillers d'orientation scolaire et professionnelle. Ce changement vient concrétiser le déplacement de leur mission de la fin de scolarité obligatoire aux classes de premier cycle du second degré (collège et premier cycle des lycées). La nature de leurs interventions voit diminuer l'examen individuel au profit des tests collectifs d'aptitudes, de connaissances ou d'intérêts dans le cycle d'observation ou la classe de troisième des collèges.

L'Inetop joue un grand rôle dans la définition de ces pratiques sous l'influence de Maurice Reuchlin qui a succédé à Mme Piéron à la tête du service de recherches dès 1950 puis à Henri Piéron à la direction de l'Institut à son départ en retraite en 1962. L'orientation a cessé d'être marginale, c'est l'âge d'or d'une psychologie expérimentale qui affine ses méthodes mathématiques avec le développement des analyses statistiques. La création de nouvelles voies scolaires se multiplie (brevet de technicien supérieur, diplôme universitaire de technologie dans l'enseignement supérieur court, diversification des baccalauréats et brevets d'enseignement professionnel liés à la poursuite au lycée de la réforme de 1963) et le service de recherche de l'Inetop, parallèlement à ses études sur les épreuves d'évaluation, développe les premières grandes enquêtes sur la scolarisation en France. Dans le même temps, le département de recherche de l'Institut pédagogique national (IPN), futur Institut national de 
recherche pédagogique (INRP), poursuit des études dans le domaine de l'orientation en se centrant sur l'observation des élèves en situation scolaire. L'intervention systématique en milieu scolaire des conseillers d'orientation scolaire et professionnelle au service de tous les élèves, si elle est reconnue et souvent appréciée, est l'objet de débats au sein même de l'institution scolaire. Le Premier ministre de l'époque, Georges Pompidou, y est opposé, affirmant que l'orientation est avant tout l'œuvre des parents et des enseignants.

\section{L'intégration de l'orientation dans l'école et le développement des activités liées à l'information}

Paradoxalement, à la fin des années 1960, le mouvement d'intégration engagé débouche sur une mise à l'écart. Un projet connu sous le nom de projet Laurent étudie une restructuration des services et une évolution de leur mission. Un des objectifs énoncés vise à tenter de régler le problème de l'information sur le monde professionnel en milieu scolaire. Il se présente comme une solution au problème de la coordination des services existants: le Bureau universitaire de la statistique (BUS) et les services d'orientation scolaire et professionnelle. C'est un plan d'ensemble qui touche les établissements et les services existants, les personnels, l'organisation hiérarchique et fonctionnelle. Le BUS est transformé en office national d'information pour l'orientation pédagogique et professionnelle (ONIOPP). Deux organismes lui sont adjoints: le Centre d'études et de recherches sur l'évolution des qualifications et l'analyse des fonctions et l'Institut national d'étude, de formation et de recherche pour l'orientation pédagogique et professionnelle par intégration de l'Inetop et transformation des autres centres de formation en centres régionaux associés. L'organisation des services au niveau académique est placée sous la responsabilité d'un chef de service académique. Ce projet prévoit un nouveau statut pour les centres d'orientation transformés en centres d'information et d'orientation. Chaque centre est dirigé par un chef d'établissement comme pour les établissements scolaires. Le personnel technique est composé de professeurs-conseillers recrutés parmi les enseignants et qui seront chargés d'intervenir dans les établissements en liaison avec les professeurs, des conseillers-psychologues chargés de l'observation des élèves, la prévention des inadaptations scolaires, l'aide psycho-pédagogique dans les établissements scolaires du primaire au collège et de conseillers en éducation professionnelle qui assureront, en plus du soutien aux professeurs-conseillers, les consultations à caractère psycho-technique.

Ce projet mis à l'étude, à titre expérimental, dans deux académies dès 1967, est l'objet de nombreuses critiques et les syndicats d'enseignants feront de son retrait un préalable aux négociations qui suivront le mouvement social de contestation de mai 1968. Le développement de la scolarisation et la multiplication des voies d'orientation possibles, la recomposition du monde du travail qui s'accompagne d'une perte de visibilité du travail rend nécessaire une évolution des services d'orientation et une attention plus grande aux politiques d'information. Si le projet Laurent est abandonné, certaines de ses dispositions sont maintenues. En 1970, l'Office national d'information sur les études et les professions (Onisep), par transformation du BUS, est créé avec comme organisme associé le Centre d'étude et de recherche sur les qualifications (Céreq). Les centres d'orientation scolaire et professionnelle sont transformés en 
centres d'information et d'orientation (CIO), marquant ainsi dans leur dénomination la place de plus en plus importante que prend l'information dans les activités des services. Le nouveau statut des personnels (décret du 21 avril 1972), s'il subit quelques modifications $d u$ fait de l'intégration des personnels du BUS, se situe dans le prolongement des statuts antérieurs par transposition des corps existants. La mission des personnels est précisée : "Les conseillers concourent sous l'autorité du directeur $\mathrm{du}$ centre d'information et d'orientation, aux diverses actions d'information et d'orientation à tous les niveaux des enseignements du second degré et des enseignements supérieurs qui entrent dans les missions desdits centres. Pour les enseignements de second degré, ils participent à l'observation continue des élèves en vue de leur adaptation et de leur orientation » (cité par Caroff, 1987, p. 221-222). Un échelon départemental apparaît avec le corps des inspecteurs départementaux de l'information et de l'orientation, l'échelon académique est renforcé par la création du service académique d'information et d'orientation (SAIO) dans le cadre du renforcement de la planification scolaire et de la mise en place de nouvelles procédures d'orientation en 1973. Ces nouvelles procédures s'articulent en trois phases distinctes : une phase d'information, une phase de dialogue entre le jeune, sa famille et l'équipe éducative, et une phase d'orientation constituée de la décision d'orientation prononcée par le conseil de classe et de l'affectation placée sous la responsabilité de l'inspecteur d'académie. Dans le même temps, une circulaire (8 juin 1971) place le problème de l'information scolaire et professionnelle dans les établissements scolaires sous la responsabilité directe du chef d'établissement et fait du conseiller d'orientation son conseiller technique. Cette circulaire, par son souci d'intégrer l'information «à l'ensemble des actions éducatives menées dans le cadre de l'établissement scolaire ", préfigure, en partie, ce que sera l'éducation à l'orientation des années à venir (circulaire du 30 septembre 1995). Dès 1969, l'Inspection générale des services d'orientation avait été rétablie, son rattachement au groupe « Vie scolaire » marquant la fin du lien fort entretenu avec l'enseignement technique depuis un demi-siècle.

Cette période de réforme des services d'information et d'orientation est étroitement liée à l'effort de rénovation des formations et correspond à une modification importante des textes législatifs concernant la formation professionnelle et continue (loi du 16 juillet 1971). Selon Caroff (1987), l'ambition de cette loi, toutes proportions gardées, rejoint celle du décret de loi du 24 mai 1938 qui avait vu l'institutionnalisation de l'orientation à une exception près: l'orientation ne constitue plus le volet indispensable de la formation professionnelle. L'orientation y est évoquée, mais de manière restrictive, essentiellement sous l'angle de la documentation et de l'information. Pour cet auteur, l'absence de disposition relative à l'orientation dans le domaine de la formation continue ouvre la porte au pluralisme et à la dispersion des efforts. Dès lors, le développement des mesures concernant la formation continue, l'orientation et l'insertion professionnelle concernera peu l'activité des services d'orientation en dehors de sollicitations limitées souvent mal vécues par les personnels quand elles leur semblent remettre en cause leur action en milieu scolaire. Des institutions nouvelles apparaissent comme les missions locales et les permanences d'accueil d'information et d'orientation (PAIO) en 1982 dans le cadre des mesures d'insertion des jeunes 16-25 ans. Les centres de bilan de compétences (CIBC) sont ouverts à partir de 1986 suivis des centres de validation des acquis plus récemment. D'autres institutions comme l'Agence nationale pour l'emploi (ANPE), créée au milieu des années 1960, voient leurs missions se recentrer sur l'orientation et délèguent de 
nombreuses prestations d'orientation à des organismes privés. Cette évolution explique la recomposition du champ de l'orientation et la répartition de ses professionnels. Dans les années 1970, les conseillers d'orientation de l'Éducation nationale représentent la quasi-totalité des professionnels de l'orientation, ils représentent un peu moins d'un quart des professionnels du domaine au début des années 2000.

\section{Conclusion}

Au début des années 1970, le paysage des services actuels d'orientation est dessiné et leur évolution est rapide : 244 centres et 1300 conseillers en 1970, un peu moins de 500 centres et annexes regroupant 4500 conseillers en 2005. En 1991, les conseillers d'orientation prennent l'appellation de conseillers d'orientation-psychologues et le recrutement en formation est ouvert aux seuls diplômés de psychologie. Cette reconnaissance, liée à l'existence de la loi de 1985 protégeant le titre de psychologue, boucle une longue histoire dont nous nous sommes efforcés de retracer les grandes étapes. Cette histoire court des premières formes appliquées de la psychologie et de ses outils, les tests, au début du $\mathrm{XX}^{\mathrm{e}}$ siècle, à la reconnaissance officielle du statut de psychologue pour les personnels qui ont en charge l'orientation des élèves et des étudiants dans l'Éducation nationale ${ }^{8}$.

À la suite de Huteau (1999), il est possible de tirer de cette histoire quelques enseignements pour approfondir la réflexion sur des questions actuelles ou pour documenter une réflexion sur la création de structures d'orientation. Nous formulerons quatre remarques rapides qui vont dans ce sens :

L'histoire de l'orientation a une dimension politique importante. Elle représente un enjeu sociétal qui concerne le monde politique et ses institutions. Son développement passe nécessairement par un certain nombre de choix et décisions qui relèvent de décisions réglementaires législatives dans le champ de l'école et du travail.

31 Le développement de l'orientation s'inscrit dans un temps long. Les différentes étapes historiques, constitutives de ce temps, ont laissé leur propre marque. Il est donc utile d'analyser leurs caractéristiques pour comprendre la nature des enchaînements successifs et éviter ainsi de reproduire les blocages qui ont pu se produire à un moment donné et ont ralenti son évolution.

32 L'histoire de l'orientation et de ses services est étroitement liée au développement de la psychologie et le métier de conseiller d'orientation est l'un des métiers les plus anciens de la psychologie. L'orientation est donc tributaire du développement des sciences psychologiques et des institutions de formation et de recherche susceptibles de supporter ce développement.

L'histoire de l'orientation et de ses pratiques est inséparable de l'histoire des organismes en charge de l'élaboration et de la production des documents d'information sur les formations et le monde du travail. La réflexion sur les structures de formation propres au domaine de l'orientation inclut la prise en compte d'une réflexion sur la création parallèle de ces organismes et de leurs missions.

Pour terminer, une dernière remarque pour noter, à la suite de Michel Huteau (1999) que l'orientation «constitue un véritable patrimoine qui mériterait peut-être d'être mieux géré» (p. 23). 


\section{BIBLIOGRAPHIE}

Binet, A. (1908). Préface. L'année psychologique, vol 14, 14, V-VI.

Bisseret, N. (1974). Les inégaux ou la sélection universitaire. Paris : Presses universitaires de France.

Briand, J.-P. (1989). L'apparition du « préapprentissage » dans les grandes villes au début du xx siècle, Emploi Formation, 27-28, 42-64.

Caroff, A. (1987). L'Organisation de l'orientation en France. Évolution des origines à nos jours. Paris : EAP éditions.

Fontègne, J. (1921). L'Orientation professionnelle et la détermination des aptitudes. Paris-Neuchatel : Delachaux \& Niestlé.

Guichard, J. (1987). Orientation, aptitude, statut de l'enfant : le discours autour de l'enfance et de l'adolescence inadaptée, Document multigraphié, CNAM-INETOP.

Huteau, M. (1999). Psychologie et société : l'évolution du rôle et des méthodes des conseillers d'orientation des années 1920 à aujourd'hui, Questions d'Orientation, 1, 13-24.

Huteau, M. \& Lautrey, J. (1979). Les origines et la naissance du mouvement de l'orientation, L'Orientation scolaire et professionnelle, 1, 3-43.

Isambert-Jamati, V. (1972). La notion d'orientation dans l'enseignement secondaire, L'Orientation scolaire et professionnelle, 1, 129-141.

Labbé, E. (non daté). Discours d'inauguration de l'Institut national d'orientation professionnelle. Recueil des discours prononcés par M. Edmond Labbé. Paris : Association française pour le développement de l'enseignement technique.

Lelièvre, C. (1990). Histoire des institutions scolaires (1789-1989). Paris : Nathan.

Martin, J. (2002). L'Orientation professionnelle en France, en Allemagne et en Grande-Bretagne dans les années 1920 : un instrument de rationalisation ?. Histoire \& Sociétés. Revue européenne d'histoire sociale, 2, 93-106.

Ouvrier-Bonnaz, R. (2010). L'histoire des chaires du CNAM concernant l'Homme au travail (1900-1945) entre production de savoirs et engagement politique. Cahiers d'Histoire. Revue d'histoire critique, 111, 99-122.

Ouvrier-Bonnaz, R. (2011). Le laboratoire de pédagogie expérimentale de la Grangeaux-Belles : préoccupation sociale et question scientifique chez Alfred Binet. Recherches \& Éducations, 5, 131-147.

Ouvrier-Bonnaz, R. (2016). Henri Piéron au 41 de la rue Gay-Lussac (19281962). Dans L. Guttierrez, J. Martin et R. Ouvrier-Bonnaz, Henri Piéron (1881-1964). Psychologie, orientation et éducation (pp. 119-129). Toulouse : Éditions Octares. $\mathbf{P i e ́ r o n , ~ H . ~ ( 1 9 4 5 ) . ~ N o t e s ~ e t ~ D o c u m e n t s . ~ O r i e n t a t i o n ~}$ scolaire et orientation professionnelle, BINOP, 11-12, 190191.

Prost, A. (1968). Histoire de l'enseignement en France de 1800 à 1967. Paris : Armand Colin.

Prost, A. (1996). Des professions à l'école : jalons pour une histoire de l'orientation en France, Vie Sociale, 5, 11-24 


\section{NOTES}

1. Voir sur ce sujet Ouvrier-Bonnaz, R. (2011). Le laboratoire de pédagogie expérimentale de la Grangeaux-Belles : Préoccupation sociale et question scientifique chez Alfred Binet, Recherches \& Éducations, 5, 131-147.

2. Document multigraphié, $7 \mathrm{p}$.

3. Journal des débats politiques et littéraires du 11 avril 1866. Consultable sur Gallica. Identifiant: ark:/12148/bpt6k4561122.

4. Voir sur cette création, Ouvrier-Bonnaz, R. (2016). Henri Piéron au 41 de la rue Gay-Lussac (1928-1962). Dans L. Guttierrez, J. Martin et R. Ouvrier-Bonnaz, Henri Piéron (1881-1964). Psychologie, orientation et éducation (pp. 119-129). Toulouse : Éditions Octares.

5. Non daté, p. 152.

6. Transformation de l'Inop en Institut d'étude du travail et d'orientation professionnelle lors de sa réinstallation au 41, de la rue Gay-Lussac après reconstruction du bâtiment et rattachement au Cnam par arrêté du 14 février 1941. Voir Ouvrier-Bonnaz, R. (2010). L'histoire des chaires du Cnam concernant l'Homme au travail (1900-1945) entre production de savoirs et engagement politique. Cahiers d'Histoire. Revue d'histoire critique, 111, 99-122.

7. Piéron H. (1945). Binop, 11-12, 190-191.

8. Décret du $1^{\mathrm{er}}$ janvier 2017 qui réunit les psychologues scolaires et les conseillers d'orientation psychologues dans un corps unique de psychologues de l'Éducation nationale. Cette création modifie de manière déterminante le paysage de l'orientation en introduisant un nouveau statut des personnels qui en sont en charge. Cette histoire sera à faire.

\section{RÉSUMÉS}

Dans cet article nous nous inscrivons dans la logique des travaux des historiens de l'éducation qui s'attachent à montrer qu'il est difficile de comprendre les particularités et les spécificités de l'orientation telle qu'elle existe en France dans ses rapports avec l'institution scolaire si on en ignore l'histoire. Nous montrerons comment l'orientation naît en France, à l'aube du xx siècle, d'une volonté des pouvoirs politiques et économiques préoccupés de la dégradation du mode d'accès à l'apprentissage et au travail relayée par les préoccupations scientifiques d'intellectuels qui se veulent au service du progrès social. L'étude des textes législatifs les plus marquants, l'histoire des institutions et de leur création, mettent en lumière comment s'est opéré le passage d'une orientation professionnelle, ponctuelle, limitée au monde du travail à une orientation continue, éducative, ancrée dans l'École.

This article we adopt the approach of historians of education who demonstrate that it is difficult to understand the particularities of vocational guidance as it exists in France in its relations with the school institution if we ignore history.

We will show how the orientation is born in France, at the dawn of the twentieth century, from the will of the political and economic powers concerned about the deterioration of the way of access to training and work, relayed by the scientific concerns of intellectuals who want to serve social progress. 
The study of the most important legislative texts highlights the way in which the transition overcame, from a one-off guidance, limited to the world of work, to a continuous educational guidance, anchored in the School.

INDEX

Keywords : training, aptitude, guidance, vocational education, psychology

Mots-clés : apprentissage, aptitude, orientation, enseignement professionnel, psychologie

\section{AUTEUR}

\section{RÉGIS OUVRIER-BONNAZ}

Groupe de recherche et d'étude sur l'histoire du travail et de l'orientation (GRESHTO) - Centre de recherche sur le travail et le développement (CRTD) - CNAM 41, rue Gay-Lussac - 75005 Paris regis.ouvrier-bonnaz@lecnam.net 